NOTICE: this pre-print is the author's version of a work that was accepted for publication in Accident Analysis \& Prevention. Changes resulting from the publishing process, such as editing, corrections, structural formatting, and other quality control mechanisms may not be reflected in this document. A definitive version was subsequently published in Accident Analysis \& Prevention, 45, 241-247 https://doi.org/10.1016/j.aap.2011.07.009 This preprint is licensed CC BY-NC-ND.

Cite this article as: Minikel, E. (2012). Cyclist safety on bicycle boulevards and parallel arterial routes in Berkeley, California. Accident Analysis \& Prevention, 45, 241-247.

\title{
Cyclist Safety on Bicycle Boulevards and Parallel Arterial Routes in Berkeley, California
}

\author{
Author: \\ Eric Minikel \\ Affiliation: \\ Department of Urban Studies and Planning \\ Massachusetts Institute of Technology \\ 77 Massachusetts Avenue \\ Cambridge, MA 02139-4307 \\ Contact: \\ Email: <ericminikel@alum.mit.edu>
}

\begin{abstract}
This study compares the safety of bicyclists riding on bicycle boulevards to those riding on parallel arterial routes in Berkeley, California. Literature on the impact of motor vehicle traffic characteristics on cyclist safety shows that high motor vehicle speeds and volumes and the presence of heavy vehicles are all detrimental to cyclist safety. This suggests that cyclists may be safer on side streets than on busy arterials. Bicycle boulevards-traffic-calmed side streets signed and improved for cyclist use-purport to offer cyclists a safer alternative to riding on arterials. Police-reported bicycle collision data and manually collected cyclist count data from bicycle boulevards and parallel arterial routes in Berkeley, California from 2003 to 2010 are used to test the hypothesis that bicycle boulevards have lower cyclist collision rates and a lower proportion of bicycle collisions resulting in severe injury. While no significant difference is found in the proportion of collisions that are severe, results show that collision rates on bicycle boulevards are two to eight times lower than those on parallel, adjacent arterial routes. The difference in collision rate is highly statistically significant, unlikely to be caused by any bias in the collision and count data, and cannot be easily explained away by self-selection or safety in numbers. This is evidence that bicycle boulevards, if properly implemented, can provide cyclists with a safer alternative to riding on arterials.
\end{abstract}

Keywords: bicycle, cyclist, collision, safety, bicycle boulevard, safety in numbers 


\section{INTRODUCTION}

Research on bicyclist safety and the built environment is relatively limited. Reynolds et al. (2009) surveyed English-language papers examining the relationship between transportation infrastructure and cyclist collisions or injuries and were able to identify only 23 papers meeting the criteria. Data are often limited-only 7 of those 23 studies based their conclusions on more than 1,000 collision or injury events.

Most of the studies cited by Reynolds et al. focus on the safety impact of purpose-built bicycle facilities such as bike lanes and cycle tracks. Less effort has gone into determining which types of streets are safer for cyclists. Yet there are good reasons to believe that quiet side streets with low motor vehicle volumes, low speeds, and a relative absence of heavy vehicles, ought to be safer for cyclists than arterial streets, which tend to have high motor vehicle volumes, high speeds and function as truck routes and public transit bus routes.

Turner, Roozenburg and Francis find, intuitively enough, that where there are more motor vehicles, there are more collisions per cyclist (Turner et al. 2006). Allen-Munley et al find that truck involvement and several factors associated with high motor vehicle speeds are correlated with more severe collisions (Allen-Munley et al. 2004). Kim et al (2006) also find that truck involvement and high speeds are correlated with severe injuries (Kim et al. 2006). Though Klop and Khattak and Allen-Munley et al both find that high traffic volume is correlated with a smaller proportion of collisions being severe (Klop, Khattak 1999; Allen-Munley et al. 2004), this does not mean that cyclists would be safer overall on busier streets, since Turner's model would predict that, compared to quiet streets, busy streets would have far more collisions to begin with. Further, those studies compared roads across an entire state (North Carolina) and city (Jersey City, $\mathrm{NJ}$ ), meaning that unobserved street design and street network variables can creep in-the high-volume streets may also be more urban in character. One of the earliest studies of cyclist safety (Kaplan 1975) found a lower collision rate for minor roads than major roads, comparing roads across the United States.

Though the literature is sparse, there is some evidence that cyclist risk is correlated with high motor vehicle volumes and speeds and with the presence of heavy vehicles. This suggests that for cyclists to use quiet side streets with low motor vehicle volumes and speeds, which are not truck or bus routes, should reduce their collision rates and severities substantially compared with traveling on major streets.

"Bicycle boulevards" purport to offer cyclists just such a quiet side street alternative. Walker et al. (2009) define bicycle boulevards as "low-volume and low-speed streets that have been optimized for bicycle travel through treatments such as traffic calming and traffic reduction, signage and pavement markings, and intersection crossing treatments." They go on to note that bicycle boulevards are often implemented as a parallel network offering close alternatives to major arterial roads. To the author's knowledge, no study has yet examined the safety of bicycle boulevards relative to arterials. Walker et al. (2009) state that "Although the safety benefits 
specifically attributed to bicycle boulevards has yet to be studied, the safety benefits of traffic calming are well documented to reduce both the frequency and severity of collisions."

The city of Berkeley, California has implemented a network of seven bicycle boulevards, shown in Figure 1, spanning the city. Most of the bicycle boulevards run directly parallel to, and just one block away from, a key arterial route. The boulevards are heavily traffic calmed with a combination of chicanes, traffic circles, traffic diverters and barriers, and speed humps. Frequent signs and pavement markings indicate to motorists and cyclists that the latter have priority and, in most places, should use the full lane.

FIGURE 1. Berkeley's seven bicycle boulevards (dashed) run parallel to key arterials (solid).

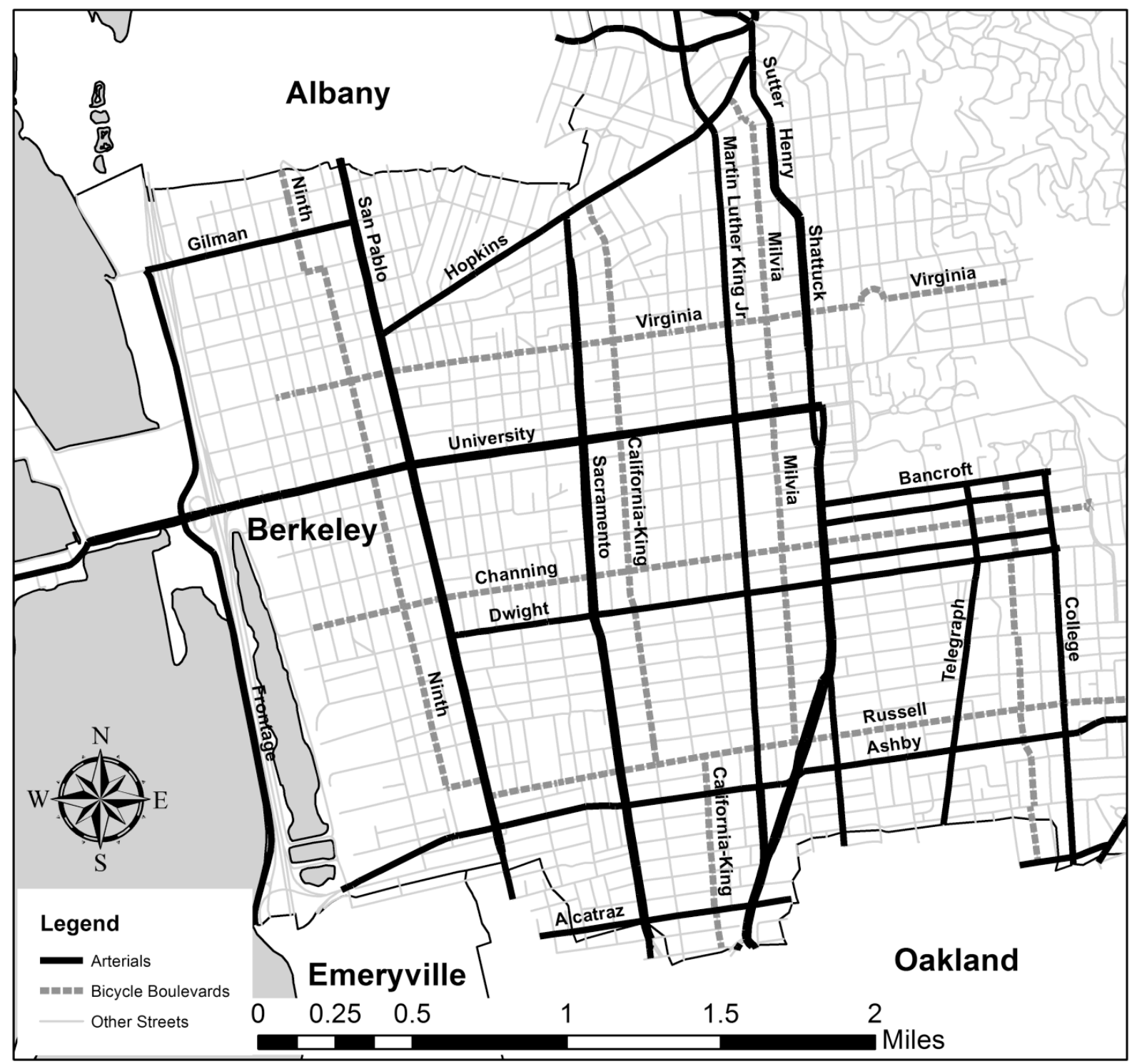

Streets: ESRI data \& maps 9.3 [electronic resource]. Redlands, CA : Environmental Systems Research Institute, Inc., c2008. City and water: ESRI StreetMap premium [electronic resource] : North America. [Redlands, Calif.] : ESRI, c2008-2009. Arterials and bicycle boulevards selected from streets or drawn manually based on City of Berkeley Bikeway Map available at http://www.ci.berkeley.ca.us/uploadedFiles/Public_Works/Level_3_-_General/Bikeway_Network.pdf 
Available data confirm that bicycle boulevards have lower motor vehicle volume than arterials (City of Berkeley Average Total Daily Traffic Volume). The City's most recent traffic counts, from 2000, found annual average daily traffic (AADT) of 5,440 for Milvia St. (a bicycle boulevard), compared to 27,599 on Shattuck Ave. and 23,502 on Martin Luther King Jr. Blvd. (arterials). Only relatively major roads were counted, so no other bicycle boulevards are included in the City's data. However, this approximately 5 to 1 difference in traffic volume is the minimum that might be expected elsewhere in Berkeley, for three reasons. First, the counts list Milvia St. as a "collector" while all other bicycle boulevards are unlabeled, suggesting that Milvia is the busiest (in terms of motor vehicle traffic) of the bicycle boulevards. Second, the counts date before bicycle boulevard implementation was completed in 2003 (City of Berkeley Bicycle Boulevard History). Third, counts taken by the author on Sacramento St. and California St. suggest motor vehicle traffic ratio on the order of 100 to 1 (Minikel 2010).

Berkeley's citywide speed limit of $25 \mathrm{mph}$ applies to all of the bicycle boulevards and arterials highlighted in Figure 1 except for Sacramento St. and San Pablo Ave. (30 mph), and part of University Ave. (35 mph), all of which are arterial streets (City of Berkeley Speed Limits). Actual speed data are not available, but the heavy traffic calming on the bicycle boulevards as documented in Minikel (2010) suggests that speeds there are likely to be lower than speeds on arterial streets.

Finally, the Alameda County Transit system map shows public bus routes on almost all of the major arterials shown in Figure 1, and none of the bicycle boulevards (Alameda County Transit City Map). Data on truck routes are not available.

It appears, then, that Berkeley's bicycle boulevards have lower motor vehicle volume and speed, and fewer heavy vehicles than its arterial routes. Based on the literature review, it is hypothesized that:

Berkeley's bicycle boulevards are safer for cyclists, both in terms of collision rate and severity, than the arterials to which they run parallel.

This study uses police-reported collision data from January 2003 to October 2008 and cyclist count data from January 2003 to March 2010 to compute collision rates and collision severity. It compares collision rates on identical-length parallel segments of bicycle boulevards and arterials, and compares collision severity across the city's entire bicycle boulevard and arterial network.

This approach compares bicycle boulevards in their entirety to arterials in their entirety. Pairs of parallel, adjacent street segments of identical length-possible alternate routes to the same destination for many cyclists-are compared, so the comparison represents a real-life choice faced by cyclists choosing where to ride. The study seeks to answer the question, "Are cyclists safer when they ride on bicycle boulevards or when they ride on arterials?" It does not seek to determine what about bicycle boulevards may make them safer. Whereas many road 
safety studies treat motor vehicle volume as a control variable, this study treats it as one of many entangled study variables_one of many possible reasons why bicycle boulevards may be safer than arterials.

Many related avenues of study would be valuable topics for future research. Comparing cyclist collision rates on the same streets before and after those streets are treated as bicycle boulevards, would make it possible to determine whether the associated design interventions improve safety. Controlling for motor vehicle volume when comparing cyclist collision rates on bicycle boulevards with those on arterial would make it possible to determine whether any difference in safety is wholly due to a difference in traffic levels or may also be due to design features, speeds, and so on. This study does not achieve either of these aims. However, the results of this study are still valuable both to cyclists choosing where to ride and to cities planning bicycle facilities. The concept of a "side street"-a relatively low volume, low speed, often residential street which runs parallel to a high volume arterial-is common to many cities. A finding that Berkeley's bicycle boulevards are safer than its arterials would at least be suggestive that the creation of bicycle boulevards on these side streets might offer safety benefits to cyclists. In order for this to be true, it would need to either be true that bicycle boulevards are safer after treatment than before, or that bicycle boulevards draw cyclists away from dangerous arterials onto already-safe side streets. This study does not examine either of these possibilities, but instead seeks to provide a first step towards the study of bicycle boulevard safety by simply showing that Berkeley's bicycle boulevards, as they exist today, are safer than parallel arterial routes.

\section{MATERIAL AND METHODS}

\subsection{Collision data}

Berkeley collision data come from the Statewide Integrated Traffic Records System (SWITRS) maintained by the California Highway Patrol (CHP). This dataset includes all traffic collisions reported to local or state traffic enforcement agencies. Of bicycle collisions, the vast majority occurred on local streets and so data come ultimately from local police reports. KABCO encoding is used, with the five severity levels defined as: fatal, injury (severe), injury (other visible), injury (complaint of pain) and property damage only. For the purposes of this study, fatal and injury (severe) collisions are considered to be "severe".

This study is based on 1715 bicycle-motor vehicle collisions occurring in Berkeley between January 2003 and October 2008, inclusive. The starting point in 2003 is chosen to correspond to the completion of bicycle boulevard implementation. (City of Berkeley Bicycle Boulevard History), and October 2008 is the last date for which data were available. Because the hypothesis of this study is based on evidence about the impact of motor vehicle traffic characteristics on cyclist safety, only bicycle-motor vehicle collisions are considered. However, Minikel (2010) conducts the same analysis including bicycle-bicycle, bicycle-pedestrian and single cyclist incidents as well, and finds very similar results. 
The collisions were geocoded and then assigned to streets of interest based on data attributes indicating the cyclist's direction of travel, movement preceding collision, and whether or not the collision occurred at an intersection.

\subsection{Exposure data}

Since 2000, the City of Berkeley has conducted sporadic counts of cyclists. These counts are collected manually, by staff or volunteers standing on street corners making tally marks on pages. As of May 2010, the city's most recent counts were in 2009. The author and another volunteer conducted nine additional counts in Berkeley in March 2010, for a total of 121 counts at 102 intersections available for this research.

Virtually all of the counts were taken during weekday PM commute hours (4:00p 6:00p), so to control for temporal variation, only PM peak counts are used in computing exposure measures. This "control" only ensures that counts on different streets are comparable; there is still a question of whether PM peak travel patterns are representative of cyclist route choice more generally, and this question is addressed in the Results section below.

Count data from January 2003 to March 2010 are used to create an exposure measure. While it is not ideal to use count data from beyond the time period for which collision data are available, the design of the bicycle boulevards was relatively static during 2008-2010, so it is believed that counts taken in 2010 are still fairly representative of cyclist traffic patterns during 2003 - 2008. Including later count data makes it possible to create exposure measures for more streets of interest, and to have enough repeated counts to be able to average out-and examine-fluctuations in counted volumes.

In each count, the number of cyclists is specified for each approach and each movement (left turn, right turn or straight). To obtain exposure measures for each street based on these intersection counts, straight-through cyclists are each counted as a full cyclist and turning cyclists as half. Using many different counts to create a single exposure measure for each street requires some way of combining or averaging the count data. A given street-take Milvia St. as an example-may have several counts, conducted over several years and at a few different cross streets, with some repetition. Here, counts are averaged first over time, so that the traffic on Milvia at Channing is considered to be the average of the traffic observed at that point in 2003, 2004, 2005, 2009 and 2010. Next they are averaged over space, so that the traffic on Milvia is considered to be the average of the traffic observed at Channing and at Hearst, the two cross-streets where Milvia has been counted. Exposure measures obtained in this manner are shown in Table 1, where "B" represents bicycle boulevards and " $A$ " represents arterials. Within each grouping of streets, the bicycle boulevard runs parallel and adjacent (or nearly so) to the arterial(s). 
TABLE 1 Exposure measures obtained for each street in Berkeley

\begin{tabular}{|l|l|l|l|l|}
\hline \multicolumn{1}{|c|}{ Street } & Type & $\begin{array}{c}\text { Average 2-hour } \\
\text { PM peak volume }\end{array}$ & $\begin{array}{c}\text { Number of } \\
\text { counts }\end{array}$ & $\begin{array}{c}\text { Number of } \\
\text { cross } \\
\text { streets }\end{array}$ \\
\hline Hillegass-Bowditch & B & 107.6 & 8 & 3 \\
Telegraph & A & 114.5 & 3 & 1 \\
College & A & & 0 & 0 \\
\hline Milvia & B & 188.1 & 10 & 2 \\
Shattuck & A & 185.0 & 1 & 1 \\
MLK & A & 44.8 & 5 & 3 \\
\hline California & B & 39.8 & 9 & 3 \\
Sacramento & A & & 0 & 0 \\
\hline 9th & B & 36.4 & 7 & 3 \\
San Pablo & A & 43.2 & 3 & 3 \\
\hline Virginia & B & 61.3 & 4 & 1 \\
University & A & 28.9 & 6 & 2 \\
\hline Channing & B & 87.2 & 13 & 6 \\
Dwight & A & & 0 & 0 \\
\hline Russell & B & 63.6 & 9 & 4 \\
Ashby & A & 29.6 & 6 & 4 \\
\hline
\end{tabular}

Unfortunately, no count data were available for three streets-College, Sacramento and Dwight. Also, only one count was included for Shattuck, but an analysis of four pre-2003 counts and a simultaneous count of Milvia and Shattuck in 2010 suggests that the ratio of cyclist traffic on Milvia to that on Shattuck is at least as high as that implied by the exposure measures in Table 1 (Minikel 2010).

\section{METHODOLOGY}

This study compares both collision rate and collision severity on bicycle boulevards and arterials.

Collision rate is computed by dividing the number of collisions observed on each street segment by the exposure measures computed as described above. Observe that this method derives the collision rate at current exposure levels; it does not provide a formula for collision rate at all levels of cyclist exposure. The effects of exposure on collision rate are addressed below. Collision rates are compared between pairs of parallel, adjacent segments of bicycle boulevards and arterials, starting and ending at the same cross streets. A risk ratio is computed by dividing the arterial's collision rate by that of the bicycle boulevard. Poisson regression is used to determine the statistical significance of this difference in risk. 
Because severe injuries are too rare to study the level of individual street pairs, collision severity is computed by dividing the total number of severe injury collisions by the total number of collisions for all arterials and all bicycle boulevards in Berkeley. A difference of proportions test is used to determine statistical significance.

\section{RESULTS}

\subsection{Collision severity}

Table 2 shows the proportion of collisions that are severe on each street type. " $A$ " represents collisions occurring on arterials, " $B$ " represents collisions on bicycle boulevards, "AB" represents collisions occurring at the intersection of an arterial and a bicycle boulevard, and "O" represents collisions occurring on other streets. The results in Table 2 show that the bicycle boulevards actually have a higher proportion of collisions that are severe than do arterials, though with a $p$ value of about .6 for a two-tailed difference of proportions test, the difference is far from being statistically significant. It appears that there is no difference between Berkeley's bicycle boulevards and arterials in the proportion of bicycle collisions that are severe.

TABLE 2 Proportion of bicycle-motor vehicle collisions that are severe on different street types in Berkeley, January 2003 - October 2008.

\begin{tabular}{|l|r|r|r|}
\hline Street type & $\begin{array}{c}\text { Number } \\
\text { of } \\
\text { Collisions }\end{array}$ & $\begin{array}{c}\text { Number } \\
\text { Severe }\end{array}$ & \multicolumn{1}{c|}{$\begin{array}{c}\text { Proportion } \\
\text { Severe }\end{array}$} \\
\hline A & 575 & 21 & $3.7 \%$ \\
B & 185 & 9 & $4.9 \%$ \\
AB & 3 & 0 & $0.0 \%$ \\
\hline & 952 & 41 & $4.3 \%$ \\
\hline
\end{tabular}

\subsection{Collision rates}

Table 3 compares the collision rates on all the streets of study in Berkeley. Bicycle boulevards ("B") are grouped with arterials ("A") they parallel. Within each grouping, a comparable street segment-starting and ending at the same cross streets-is considered. The collisions column indicates the total number of bicycle-motor vehicle collisions that occurred on the relevant street segment between January 2003 and October 2008, inclusive. This figure is available for all streets. The exposure column indicates the average two-hour PM peak count-averaged first over time and then over space, as described above. Some streets had no count data available. For those with count data, the relative collision rate listed in Table 3 is the quotient of the two 
preceding columns. Note that the units of the relative collision rate column are not meaningfulcollisions in about six years, divided by cyclists in two hours. Also, each bicycle boulevard is comparable only to the arterial(s) immediately following it in the table; streets from different groups are not comparable because the length of street segments may be different.

TABLE 3 Collisions, exposure and relative collision rate for Berkeley streets based on January 2003 - October 2008 bicycle-motor vehicle collision data and $2003-2010$ exposure data.

\begin{tabular}{|c|c|c|c|c|}
\hline Street Name & Street & Collisions & Exposure & Relative collision rate \\
\hline Hillegass-Bowditch & $B$ & 5 & 107.6 & 0.05 \\
\hline Telegraph & $A$ & 21 & 114.5 & 0.18 \\
\hline College & $A$ & 30 & & \\
\hline Milvia & $B$ & 26 & 188.1 & 0.14 \\
\hline Shattuck & $A$ & 47 & 185 & 0.25 \\
\hline MLK & A & 18 & 44.8 & 0.40 \\
\hline California & $B$ & 9 & 39.8 & 0.23 \\
\hline Sacramento & $A$ & 15 & & \\
\hline Ninth & $\bar{B}$ & 3 & 36.4 & 0.08 \\
\hline San Pablo & $A$ & 26 & 43.2 & 0.60 \\
\hline Virginia & B & 6 & 61.3 & 0.10 \\
\hline University & $A$ & 17 & 28.9 & 0.59 \\
\hline Channing & $B$ & 19 & 87.2 & 0.22 \\
\hline Dwight & $A$ & 11 & & \\
\hline Russell & $B$ & 7 & 63.6 & 0.11 \\
\hline Ashby & $A$ & 26 & 29.6 & 0.88 \\
\hline
\end{tabular}

For each and every pair of bicycle boulevard and arterial where count data are available, the bicycle boulevard has the lower collision rate. The risk ratio-collision rate on the arterial divided by collision rate on the bicycle boulevard-ranges from 1.8 for Shattuck v. Milvia to 8.0 for Ashby v. Russell.

The statistical significance of these differences in risk is tested using a Poisson regression on each street pair. This approach assumes that bicycle-motor vehicle collisions occur as the result of a Poisson process over cyclist trips, with each street's Poisson process having its own parameter indicating the frequency of collisions. For each street pair, Poisson regression asks the question, "If this arterial $(A)$ and this bicycle boulevard $(B)$ actually had the same underlying parameter, what is the probability that one would happen to observe a difference in collision rates at least as large as that shown in Table 3?"

To apply this regression, a subtotal of collisions is computed for each street in each year studied. For instance, Hillegass-Bowditch had a total of 5 collisions over 2003-2008-2 in 2003, 
1 in 2004, 1 in 2005, 0 in 2006, 1 in 2007 and 0 in 2008. The exposure measures shown for each street in Table 3 are used for each year, with an adjustment for 2008 containing only 10 months of data. Because these exposure measures reflect cyclist volume in a 2-hour period, a multiplier of 750 is applied to translate these into estimated yearly cyclist volumes. This figure is based on the conservative assumption that PM peak represents $1 / 3$ of daily volume and that there are 250 weekday equivalents in a year. The exact number chosen does not impact the results of the regression, but some multiplier is necessary in order to respect the "rare event" nature of Poisson processes. If, for instance, cyclists on Ashby actually experienced 26 collisions for every 30 trips, then a Poisson model might not be appropriate.

The Poisson regression model used is shown in Equation 1. Here, exposure is an offset, so its coefficient is constrained to be 1 . $a$ and $b$ are parameters to be fitted (they do not represent arterials and bicycle boulevards). bblvd is a boolean variable indicating whether each street is a bicycle boulevard. For each bicycle boulevard-arterial pair, $e^{-b b l v d}$ will represent the risk ratio. The purpose of the Poisson regression is not to determine this risk ratio, which can be achieved more simply by dividing the numbers in the "relative collision rate" column of Table 3 , but to determine how statistically significant bblvd is.

\section{EQUATION 1 Poisson regression model with exposure offset}

$$
\ln (\text { collisions })=\ln (\text { exposure })+a+b^{*} b b / v d+\varepsilon
$$

Table 4, below, shows a sample of the data on which this Poisson model was fitted. The first two columns are shown for explanatory purposes; only the columns collisions, exposure and bblvd were used in the model. This sample is for comparing Ninth St. (a bicycle boulevard) with San Pablo Ave. (an arterial). Similar tables were developed for each pair of streets to be compared. In the case of Ninth St., pairs of years were grouped together because the natural log function in Equation 1 will not tolerate rows with zero collisions. 
TABLE 4 Sample of data to which Poisson regression was applied

\begin{tabular}{|c|c|c|c|c|}
\hline Street & $\begin{array}{l}\text { Time } \\
\text { period }\end{array}$ & collisions & exposure & bblvd \\
\hline \multirow{3}{*}{ 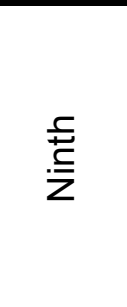 } & $\begin{array}{l}2003- \\
2004\end{array}$ & 1 & 54650 & 1 \\
\hline & $\begin{array}{l}2005- \\
2006\end{array}$ & 1 & 54650 & 1 \\
\hline & $\begin{array}{l}2007- \\
2008\end{array}$ & 1 & 50096 & 1 \\
\hline \multirow{6}{*}{$\begin{array}{l}\frac{0}{0} \\
\frac{0}{\pi} \\
\frac{1}{\infty} \\
\tilde{\varpi} \\
\tilde{c}\end{array}$} & 2003 & 2 & 32375 & 0 \\
\hline & 2004 & 1 & 32375 & 0 \\
\hline & 2005 & 4 & 32375 & 0 \\
\hline & 2006 & 5 & 32375 & 0 \\
\hline & 2007 & 10 & 32375 & 0 \\
\hline & 2008 & 4 & 26979 & 0 \\
\hline
\end{tabular}

Table 5 shows the results of the Poisson regression described above. The third column, risk ratio, shows the ratio of the collision rate on the arterial to that on the neighboring bicycle boulevard, and the final column shows the likelihood that the difference in observed collision rate during 2003-2008 could come about by chance if in fact the two streets had identical underlying collision rates. Table 5 shows that the differences observed are both large and statistically significant for all street pairs examined.

TABLE 5 Risk ratio and statistical significance of Poisson regressions on street pairs in Berkeley

\begin{tabular}{|l|l|c|r|}
\hline Arterial & Bicycle Boulevard & $\begin{array}{r}\text { Risk } \\
\text { ratio }\end{array}$ & p value \\
\hline Telegraph & $\begin{array}{l}\text { Hillegass- } \\
\text { Bowditch }\end{array}$ & 3.9 & 0.0058 \\
\hline Shattuck & Milvia & 1.8 & 0.0128 \\
\hline MLK & Milvia & 2.9 & 0.0005 \\
\hline $\begin{array}{l}\text { San } \\
\text { Pablo }\end{array}$ & Ninth & 7.3 & 0.0011 \\
\hline University & Virginia & 6.0 & 0.0002 \\
\hline Ashby & Russell & 8.0 & $<0.0001$ \\
\hline
\end{tabular}

If all types of bicycle crashes rather than only bicycle-motor vehicle collisions are included, the risk ratios range from 1.5 to 7.0 and all are statistically significant at the .01 level or better with the exception of Milvia St. vs. Shattuck Ave., whose difference is still nearly significant with a p value of .054 (Minikel 2010).

As mentioned above, the Poisson regression assumes that collision and count data available for this study are representative of total collisions and total exposure. Because this 
assumption may not be correct, a separate discussion of the reliability of these measures follows.

\subsection{Reliability of data}

\subsubsection{Collision data}

Reported collisions could be an unreliable measure of total collisions if the streets being compared have different populations with different levels of inclination to report collisions. Though the author did not notice any categorical demographic difference in cyclists on the two street types while observing cyclists in Berkeley, hard data to address this concern are unavailable. However, a quick sensitivity analysis is worthwhile. Consider Milvia vs. Shattuck, the bicycle boulevard-arterial pair with the smallest difference in estimated collision rate. Even for this pair, in order to invalidate the conclusion that the bicycle boulevard is safer, it would have to be true that reporting rates on the arterial are higher, and by almost a factor of two. So if Milvia had a reporting rate of $40 \%$, as one study has found for bicycle crashes (Moritz 1997), Shattuck would need a reporting rate of almost $80 \%$. Given that the profile of cyclists on the two street types is not so obviously different, this is somewhat hard to imagine.

It is also possible that a difference in the proportion of collisions that are severe drives a difference in reporting rates, but this would not alter the conclusion that bicycle boulevards are safer than arterials. It would simply mean that some of the difference in safety is caused by an underlying difference in severity which is masked among reported collisions.

\subsubsection{Exposure data}

The number of cyclists counted on a given street may vary at different points along the street and also by time of day, day of week, season and weather, and perhaps by year if cycling is on the rise or decline. For these reasons, a handful of counts taken at two or three cross streets over several years at weekday PM peak may be considered a poor basis for estimating the total cyclist exposure that gave rise to observed collisions. However, what are compared here are relative collision rates for pairs of parallel, adjacent streets that function as alternatives to one another for reaching the same set of destinations. The variability in cyclist volume in Berkeley is a concern only if cyclists' route choice changes over time and space. If, on the other hand, the numbers of cyclists on Street $A$ and Street $B$ rise and fall in unison, with about the same proportion choosing A over B regardless, then cyclist counts provide a good basis for estimating exposure and computing collision rates.

To assess how well the cyclist count data represent true exposure, three questions are posed:

1. How consistent is the ratio of counts on $A$ to $B$ at different points along the two streets?

2. How consistent is the ratio of counts on $A$ to $B$ over different years? 
3. How well does the ratio of A's weekday PM peak traffic to B's represent the ratio over the whole week?

An extensive treatment of these questions is given by Minikel (2010). In terms of spatial variation (Question 1), the two bicycle boulevard-arterial pairs with enough data are examined, and the variation in cyclist volume ratios over different cross streets is found to be small relative to the difference in collision rate observed.

For yearly variation (Question 2), the ratio of north-south to east-west cyclist traffic at intersections is found to be relatively consistent over different years. This does not prove that the same is true of parallel streets, but it supports the theory that cyclist route choice is consistent over time.

Regrettably, only limited data are available to address variation within the week (Question 3). The handful of available weekday AM peak counts suggest that cyclist route choice is roughly the same in the morning and evening peak, but no data are available on midday, evening or weekend travel patterns, and these could well be different since cyclists' destinations at those times are likely different than those during rush hour. Nevertheless, given the rather large risk ratios observed for some bicycle boulevard-arterial pairs, off-peak travel patterns would have to be quite different from peak (and off-peak volume quite high) in order to invalidate the conclusions reached here.

\section{DISCUSSION}

If one can be reasonably certain that the results in Table 3 reflect a real and significant difference in collision rate between bicycle boulevards and arterials, there is still room to ask what drives the difference. The hypothesis of this paper is based on literature findings about motor vehicle speed and volume and the presence of heavy vehicles, so it expects that a difference in these motor vehicle traffic characteristics is what creates the difference in safety for cyclists. However, there are other possible explanations for the observed difference in safety between bicycle boulevards and arterials which must be addressed.

One is self selection: perhaps arterials simply attract more risk-taking cyclists, while bicycle boulevards attract more safety-conscious cyclists. Yet of the 1715 bicycle-motor vehicle collisions in the Berkeley dataset, police found the cyclist at fault in just $705(41 \%)$. The motorist was found at fault in 925 collisions (54\%) and the remaining 85 (5\%) were no-fault. While this simple either-or assignment of fault does not tell the whole story of what causes a collision, these numbers are nonetheless hard to square with the idea that cyclist behavior wholly determines risk level, which is necessary in order for self-selection alone to create the apparent difference in collision rate.

Another concern relates to the relationship between exposure and collision rate. What have been computed here are collision rates at current levels of cyclist exposure. Collision rates might well be different at different levels of cyclist exposure. In particular, it might be 
hypothesized that bicycle boulevards appear safer only because they carry a greater number of cyclists, thus achieving "safety in numbers." The theory of causative safety in numbers, as elaborated by researchers such as Jacobsen (2003) and Robinson (2005), holds that the presence of cyclists causes drivers to behave differently, thus making cyclists safer. In fact, this theory cannot explain away the difference in collision rates found here. Figure 2 plots risk per cyclist versus exposure for each street. Risk per cyclist used here is an adjusted relative collision rate: to make all the streets at least roughly comparable to one another, relative collision rates from Table 3 are divided by the length of street segment considered for each street. Volume, again, is simply the average two-hour PM peak count.

FIGURE 2 Collision rate vs. exposure for arterials (A) and bicycle boulevards (B) in Berkeley

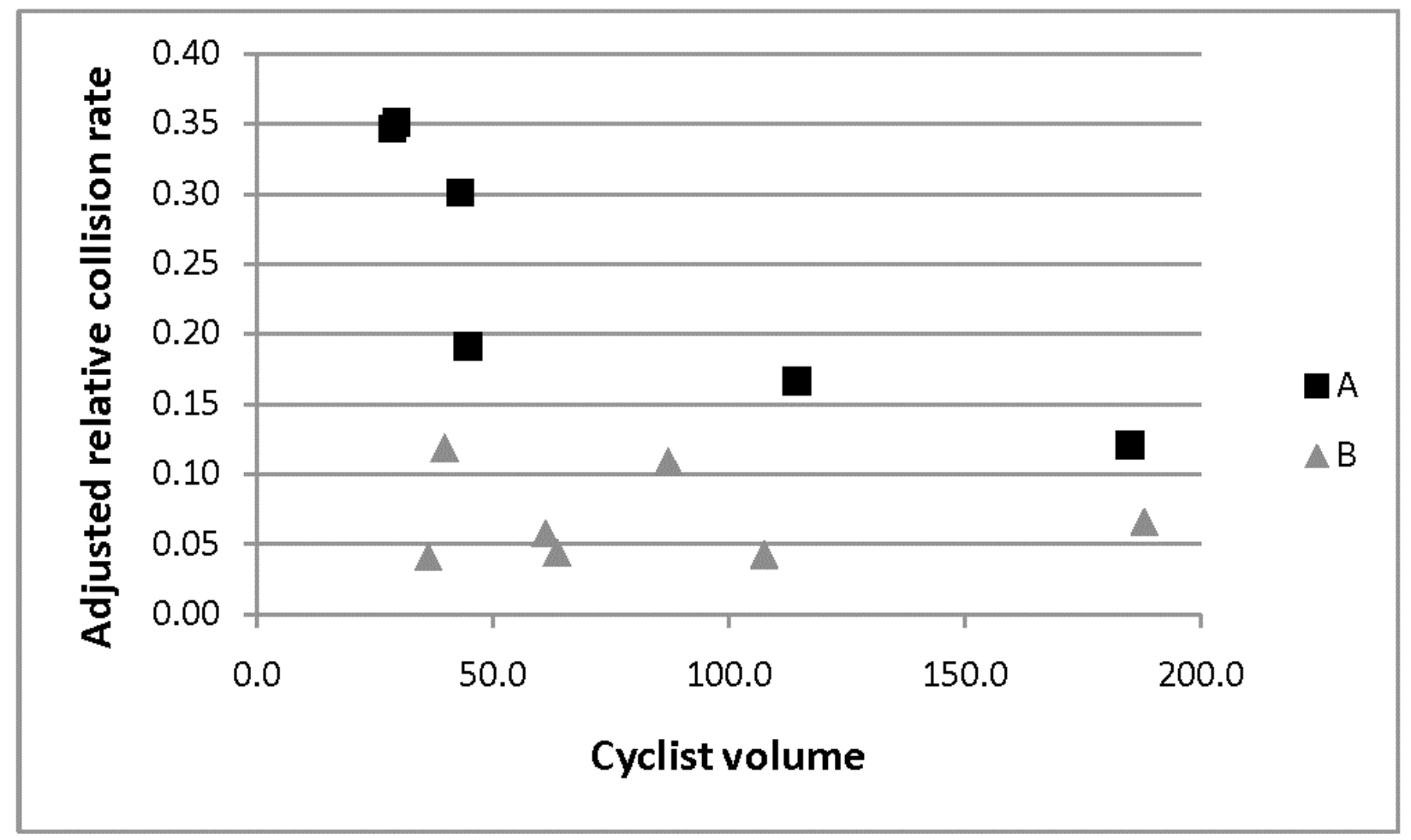

The results overall are reasonably consistent with correlative safety in numbers: high collision rates on (some) low cyclist volume streets, low collision rates on high cyclist volume streets. Yet the different symbols used for different street types helps to address causation: do cyclists ride on streets that they (correctly) believe are safer for them, or does the presence of cyclists make a street safer for cyclists? Figure 2 suggests that street type (bicycle boulevard versus arterial) does matter for cyclist safety, because at every point along the x-axis, the arterials have higher collision rates than the bicycle boulevards. Alongside that trend, it also appears that, for arterials at least, those with higher cyclist volume are safer for cyclists. Still, causation could flow in either direction. The two black squares towards the right represent Telegraph Ave. and Shattuck Ave. Perhaps the large number of cyclists there makes cycling safer, or perhaps the sharrows and intermittent bike lanes on Telegraph, and the low speeds and frequent stoplights on Shattuck are what make cycling safer, and thus draw more cyclists. 
In sum, Figure 2 does not refute the idea that cyclist numbers contribute to cyclist safety, but it shows that, at a minimum, street type-bicycle boulevard versus arterial-also matters. The results shown above, that bicycle boulevards have lower bicycle-motor vehicle collision rates than arterials, cannot be explained away by safety in numbers.

\section{CONCLUSIONS}

Berkeley has created, as the centerpiece of its bicycle plan, a network of seven bicycle boulevards - traffic-calmed side streets designated and improved for bicycle use-that stretches over the city. Compared to the arterials that they parallel, the bicycle boulevards have low motor vehicle volumes and speeds and few heavy vehicles. Using police-reported collision data and the city's cyclist count data, this study finds that Berkeley's bicycle boulevards do indeed have lower collision rates for cyclists than their parallel arterial routes. This is true for all six bicycle boulevard-arterial pairs for which data are available, with risk ratios ranging from 1.8 to 8.0. This is true whether only reported bicycle-motor vehicle collisions or all reported bicycle crashes are examined. Poisson regression reveals that the difference in collision rate is highly statistically significant. Safety in numbers cannot entirely explain the observed safety differences, because at all levels of cyclist exposure, bicycle boulevards have a lower collision rate than arterials.

No significant difference in collision severity is found between the two street types. This may reflect a threshold of severity needed in order for a collision to be reported, which would mean that the difference in total risk of severe injury is actually comprised of a smaller difference in collision rate than that observed, multiplied by some unobserved difference in the proportion severe. Either way, it is true that for cyclists, bicycle boulevards carry a lower overall severe injury risk than arterials.

The results of this study demonstrate that cyclists are safer when riding on Berkeley's bicycle boulevards than on parallel arterial routes. Of course, these bicycle boulevards represent a package of characteristics-lower motor vehicle volumes, lower speed and a relative absence of heavy vehicles as well as differences in land use, position within the road network, and a host of design elements and traffic calming features. This study does not control for any of these variables and makes no attempt to determine which of these elements brings about the observed difference in safety.

The streets considered in this study would be appropriate subjects for several related avenues of research. A before-after study of collision rates on the bicycle boulevards before and after 2003 could help to isolate the effect of the design interventions undertaken as part of the City of Berkeley's bicycle plan. A study with richer motor vehicle volume and speed data could isolate the contribution of each of these variables to the observed difference in overall cyclist safety between bicycle boulevards and arterials. 
Despite its limitations, the comparison of parallel routes made here represents the reallife choice between two routes to the same destination, each route with its own package of characteristics. This is a choice that cyclists face in choosing where to ride, and it is also a choice that planners face in choosing where to encourage cycling. Therefore the result that Berkeley's bicycle boulevards are safer than its arterials is useful even without knowing why they are safer. It is imaginable, for instance, that the difference in cyclist safety observed here is almost entirely due to a difference in motor vehicle volumes between bicycle boulevards and arterials, with almost no difference attributable to the traffic calming and design features added when the City of Berkeley branded the streets as bicycle boulevards. Even if that is true, the results of this study still suggest that cyclists would do well to ride on those bicycle boulevards and that other cities would do well to consider encouraging cyclists to use bicycle boulevards. While those cities must take many issues into account in any bicycle facility planning process, the two to eightfold difference in collision rate found here is surely one factor worthy of consideration. 


\section{REFERENCES}

Allen-Munley, C., Daniel, J., Dhar, S., 2004. Urban bicycle route safety rating logistic model. Transportation Research Record 1878, 107-115.Jacobsen, P.L., 2003. Safety in numbers: more walkers and bicyclists, safer walking and bicycling. Injury Prevention 9, 205-209.

Kaplan, J. 1975. Characteristics of the regular adult bicycle user. MSc thesis, Civil Engineering Department, University of Maryland.

Kim, J.K., Kim, S., Ulfarsson, G.F., Porrello, L.A., 2006. Bicyclist injury severities in bicyclemotor vehicle accidents. Accident Analysis and Prevention 39 (2), 238-251.

Klop, J.R., Khattak, A.J., 1999. Factors Influencing Bicycle Crash Severity on Two-Lane, Undivided Roadways in North Carolina. Transportation Research Record 1674, 78-85.

Minikel, E., 2010. Street Typology and Bicyclist Safety: A Systems Approach. Massachusetts Institute of Technology, Master of Science Thesis.

Moritz, W.E., 1997. Survey of North American Bicycle Commuters: Design and Aggregate Results. Transportation Research Record 1578, 91-101.

Reynolds, C.C.O., Harris, M.A., Teschke, K., Cripton, P., Winters, M. 2009. The impact of transportation infrastructure on bicycling injuries and crashes: a review of the literature. Environmental Health 8(47).

Robinson, D.L., 2005. Safety in numbers in Australia: more walkers and bicyclists, safer walking and bicycling. Health Promotion Journal of Australia 16(1).

Turner, S.A., Roozenburg, A.P., Francis, T., 2006. Predicting Accident Rates for Cyclists and Pedestrians. Land Transport New Zealand Research Report 289.

Walker, L., Tressider, M, Birk, M., 2009. Fundamentals of bicycle boulevard planning \& design. Prepared for the Portland State University Initiative for Bicycle and Pedestrian Innovation. 


\section{WEB REFERENCES}

City of Berkeley. Bicycle Boulevard History.

http://www.ci.berkeley.ca.us/ContentDisplay.aspx?id=6748

Undated. Accessed July 30, 2010.

City of Berkeley. Average Total Daily Traffic Volume.

http://www.ci.berkeley.ca.us/uploadedFiles/Public_Works/Level_3_-_General/TrafficVolumeMaj or_Collector.pdf

Undated. Accessed May 9, 2010.

City of Berkeley. Speed Limits.

http://www.ci.berkeley.ca.us/ContentDisplay.aspx?id=8244

Undated. Accessed May 15, 2010.

Alameda County Transit. 2010. City Map.

http://www.actransit.org/pdf/maps/version_14/city_map.pdf

Accessed May 23, 2011. 